\section{Regulation of BRCA1}

SIR - BRCA1, a gene conferring susceptibility to early onset familial breast and ovarian cancer, has recently been cloned ${ }^{1}$ We have found that the $5^{\prime}$ end of $B R C A I$ lies head to head with the $5^{\prime}$ end of the $1 A 1-3 B$ gene $^{2}$, with a maximum distance of 295 base pairs (bp) between their putative first exons. This raises the possibility

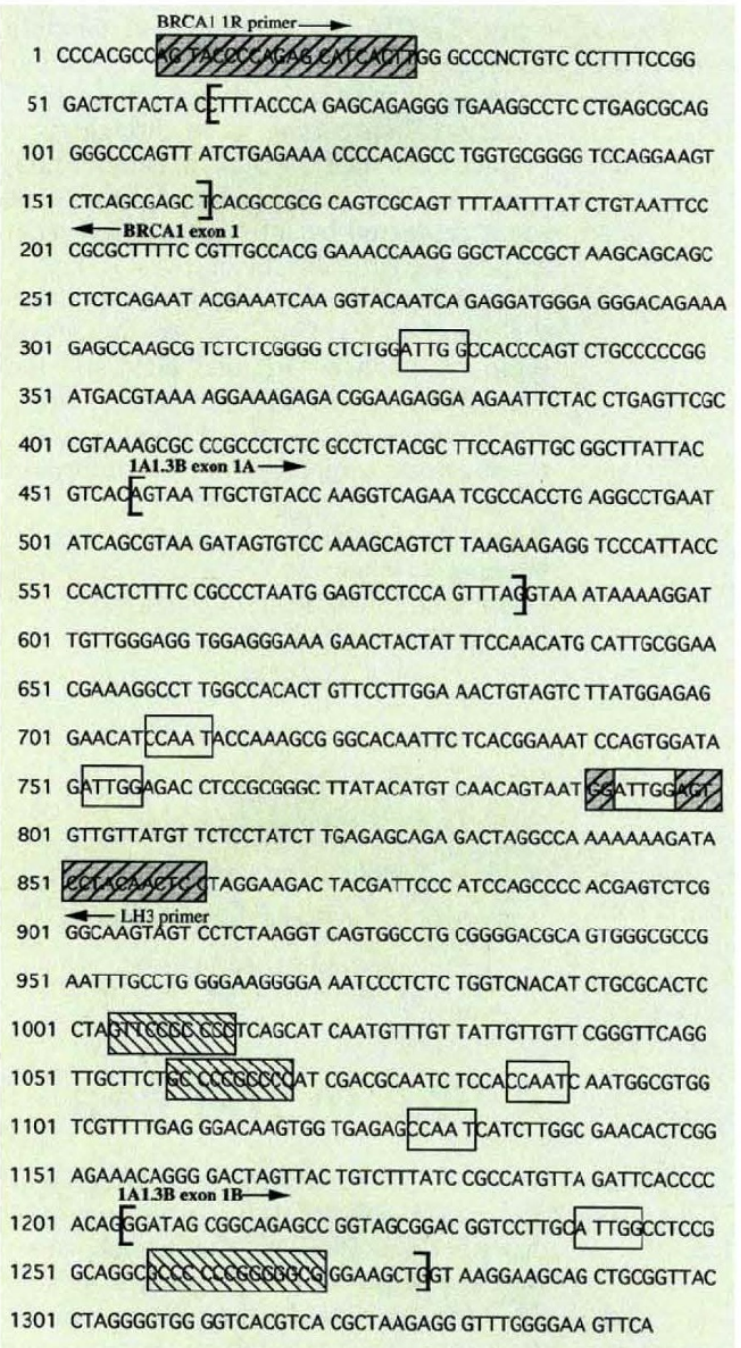

Sequence analysis of the region between the BRCA1 and $1 A 1$ $3 B$ genes. Brackets, exon sequences; hatched boxes $Z D$, positions of primers BRCA1 1 R and LH3; open and hatched boxes $\Pi \square$, positions of CAT and GC boxes, respectively.

of coregulation of these two genes by a bidirectional promoter and the potential involvement of $1 A 1-3 B$ in breast and ovarian tumorigenesis.

During the search for $B R C A 1$, we isolated a number of candidate genes ${ }^{3,4}$. One of these, $1 A 1-3 B$, was isolated from a complementary DNA expression library with antisera to the ovarian cancer marker CA125, and was characterized in our laboratories $^{2}$. 1A1-3B maps to the same $\mathrm{P} 1$ artificial chromosome (PAC) clone as
$B R C A 1$, and the two genes are divergently expressed ${ }^{4}$.

The genomic sequence at the $5^{\prime}$ end of the $1 A 1-3 B$ gene was cloned and partially sequenced. This sequence contained the previously detected first exon of $1 A 1-3 B$ (ref. 2) as well as $800 \mathrm{bp}$ of new sequence upstream. Comparison of this sequence the database revealed $100 \%$ identity to a $131 \mathrm{bp}$ region, $600 \mathrm{bp} 5^{\prime}$ to our published first exon, with a randomly isolated messenger RNA from the myeloblast cell-line KG1, termed ORF (N. Nomura et al., unpublished results; accession number D30756). ORF also contained sequences identical to exons 2-19 of $1 A 1-3 B$, but not the previously published exon 1 (here named exon 1B). This suggests that an alternative $5^{\prime}$ exon (here named exon 1A), not present in the original $1 A 1$ $3 B$ cDNA clone, is expressed in KG1 cells. This provides evidence for alternative $5^{\prime}$ starts of transcription in the $1 A 1-3 B$ gene.

Southern analysis of $B R C A 1$ - and $1 A 1-3 B$-containing PAC and cosmid clones $^{4}$ revealed that the putative first two exons of $B R C A I$ and the $5^{\prime}$ region of 1A1-3B mentioned above (as well as a portion of the 1A1-3B DNA data not shown) all reside on a single 3-kilobase (kb) PsttI fragment. Polymerase chain reaction (PCR) analysis detected a single 750-bp fragment, with the primer pair $1 \mathrm{R}$ and $\mathrm{LH} 3$ (see figure). This indicated that $B R C A 1$ exon 1 is centromeric to the $1 A 1-3 B$ gene and that the distance between the two is less than $300 \mathrm{bp}$. To confirm this result, the PCR product and cosmid A11100 were sequenced with primers $1 \mathrm{R}$ and LH3. The sequence from these primers overlapped and the 1R-primed sequence read directly into the $5^{\prime}$ region of the $1 A 1-3 B$ gene. This result mapped the distance between the two genes as less than $295 \mathrm{bp}$ (see figure). Analysis of the $1 A 1-3 B$ gene 5 region, and the sequence between the putative first exons of $1 A 1-3 B$ and $B R C A 1$, for potential promoter or enhancer sites revealed seven potential
'CAT' boxes as well as other motifs (see figure). No sequences likely to correspond to a TATA box were found.

The proximity of these two genes raises the possibility of shared promoter and/or enhancer sequences and thus coregulation of expression. There are a number of examples of such bidirectional promoters, including those directing the expression of the $\alpha 1$ and $\alpha 2$ collagen genes ${ }^{5}$, of the dihydrofolate reductase gene and the human homologue of the bacterial MutS gene $^{6}$, of Surf-1 and Surf-2 (Ref. 7) and the Wilms' tumour WTI and Wit-1 genes $^{8,9}$. Recent work on the collagen genes provides clear evidence for a bidirectional promoter involving non-TATA elements both between and within the exons of both genes, all of which are critical for the optimal and coordinated expression of both genes ${ }^{5}$. It is therefore conceivable that the 'CAT' boxes and other motifs found between the 1A1-3B and $B R C A 1$ genes are important for the expression of both $1 A 1-3 B$ and $B R C A 1$.

A model of dis-coordinate expression of $B R C A 1 / 1 A 1-3 B$ would predict a decrease of $B R C A 1$ expression with the increased expression of $1 A 1-3 B$, as is seen in ovarian cancers. Alternatively, BRCA1 expression could be quenched by antisense RNA were an additional $5^{\prime}$ exon to lie embedded within the $1 A 1-3 B$ gene. Both mechanisms would lead to the effective downregulation of $B R C A 1$, and would remain consistent with a tumour- suppressor model for this gene.

Melissa A. Brown

Hans Nicolai

Chun-Fang $X_{u}$

Beatrice L. Griffiths

Karen A. Jones, Ellen Solomon*

Somatic Cell Genetics Laboratory

Louise Hosking

John Trowsdale

Human Immunogenetics Laboratory,

Imperial Cancer Research Fund,

Lincoln's Inn Fields,

London WC2A 3PX, UK

Donald M. Black

\section{Robert McFarlane}

Beatson Institute for Cancer Research,

Garscube Estate,

Switchback Road,

Bearsden, Glasgow G61 1BD, UK

*To whom correspondence should be addressed.

1. Miki, Y, et al. Science 266, 66-71 (1994).

2. Campbell, I.G. et al. Hum. molec. Genet. 3, 589-594 (1994).

3. Jones, K.A., et al. Hum. molec. Genet. 3, 1927-1934 (1994).

4. Brown, M.A. et al. Proc. natn. Acad. Sci. U.S.A. (in the press).

5. Heikkila, P., Soininen, R. \& Tryggvason, K. J. biol. Chem. 268, 24677-24682 (1993)

6. Shinya, E. \& Simada. T. Nucleic Acids Res. 22, 2143-2149 (1994)

7. Lennard, A. C. \& Fried, M. Molec. Cell Biol. 11, 1281-1294 (1991).

8. Campbell, C.E. et al. Oncogene 9, 583-595 (1994).

9. Eccles, M.R., Grubb, G., Ogawa, O., Szeto, J. \& Reeve, A.E. Oncogene 9, 2059-2063 (1994). 